\title{
MOVING THE GOALPOSTS: SUBJECTIVE PERFORMANCE BENCHMARKS AND THE AUMANN-SERRANO MEASURE OF RISKINESS
}

\author{
CHRISTOPHER J. BENNETT AND BRENNAN S. THOMPSON ${ }^{\dagger}$
}

\begin{abstract}
This paper parameterizes the well-known Aumann and Serrano (2008) measure to explicitly allow the riskiness of financial instruments to be examined relative to benchmark levels of return other than zero. Using real data, we demonstrate that the ordering of two financial instruments in terms of their relative riskiness may change even with only small changes in the benchmark level of return. We then show that one asset may be (uniformly) riskier than another in the sense that every member of this generalized class ranks the two assets in the same way, and that this occurs only when the Laplace transforms of the assets can be ordered over a compact subset of the positive real line. A statistical test for this Laplace order is proposed, and a simulation study shows that this test is reliable in finite samples. Finally, the proposed methodology is illustrated using data on global stock index returns.
\end{abstract}

†Corresponding author. Email: brennan@ryerson.ca Revised: October 8, 2014. 


\section{INTRODUCTION}

Aumann and Serrano (2008) define the riskiness of a financial instrument as the reciprocal of the level of absolute risk aversion at which an individual with constant absolute risk aversion (CARA) is indifferent between investing $\$ 1$ in that financial instrument and holding that same $\$ 1$ in a noninterest-bearing account. ${ }^{1}$ While Aumann and Serrano (2008) suggest that their measure depends only on the return distribution of the risky financial instrument, it can also be seen to be highly dependent on a subjective parameter, namely the assumed risk-free rate of return on the outside option (i.e., the option associated with not investing in the risky financial instrument), which they implicitly set equal to zero.

In this paper, we parameterize the Aumann and Serrano (2008) measure to make this dependence explicit. This gives rise to a class of measures, each member of which may be viewed as measuring the riskiness of financial instruments relative to a given risk-free rate of return ("benchmark"). We then consider the robustness of a riskiness ordering to changes in the benchmark. In particular, we study a restricted version of the Laplace order, ${ }^{2}$ which is a stochastic ordering that is weaker than second-order stochastic dominance, yet sufficient to guarantee that a riskiness ordering is invariant to the (subjective) choice of benchmark.

To make this result operational, we propose a statistical test for the (restricted) Laplace order, which enables users to infer riskiness orderings that are robust to the

\footnotetext{
${ }^{1}$ Aumann and Serrano (2008) define the riskiness of generic gambles, with investments in financial instruments being merely special cases.

${ }^{2}$ The Laplace order is sometimes referred to in the literature as infinite order stochastic dominance or completely monotone stochastic dominance. See Whitmore (1989), Thistle (1993), and Bennett (2008) for a review of this stochastic order and its application to problems of choice under risk.
} 
choice of benchmark in the same way that statistical tests for stochastic dominance enable users to infer orderings over gambles that are robust to the specification of preferences. ${ }^{3}$ Our test extends in various directions the paper by Knight and Satchell (2008), in which the authors test a set of necessary conditions for Laplace dominance. Specifically, the procedure developed herein (i) allows for general forms of dependence (mutual and serial); (ii) allows for the range of dominance to be determined endogenously; and (iii) tests the full set of restrictions implied by (restricted) Laplace dominance.

The outline for the paper is as follows. In Section 2, we formally define the Aumann and Serrano (2008) class of measures, and provide the condition under which the orderings induced by these measures are invariant to the choice of benchmark. Next, in Section 3, we formalize the inference problem suggested by this invariance condition and develop a resampling-based test procedure. The large sample properties of the proposed test are also examined here. Section 4 presents simulation results, followed by an empirical illustration in Section 5. Section 6 concludes. Proofs of all theoretical results are given in the Appendix.

\section{MeAsures of Riskiness}

2.1. Preliminaries. Let $\mathcal{U}$ denote the collection of strictly increasing and concave von Neumann-Morgenstern utility functions that are defined over the positive real

\footnotetext{
${ }^{3}$ There is a large and growing literature on statistical inference for stochastic dominance; see, for example, Anderson (1996), Davidson and Duclos (2000), Barrett and Donald (2003), Linton, Maasoumi and Whang (2005), Horváth, Kokoszka and Zitikis (2006), Linton, Song and Whang (2010), Bennett (2013), and references therein.
} 
line, and let $\mathcal{G}$ denote the set of financial instruments - that is, a collection of risky assets whose gross rates of return are modeled as non-negative random variables. ${ }^{4}$

An agent with utility function $u \in \mathcal{U}$ and wealth level $w>0$ is given the option to invest $\$ 1$ either in $X \in \mathcal{G}$ or an interest-bearing account guaranteeing a gross rate of return $\beta .^{5}$ As an expected utility maximizer, he prefers the $\$ 1$ investment in $X$ whenever the expected utility premium,

$$
\pi_{u}(w ; X, \beta)=\mathbf{E}[u(w-1+X)]-u(w-1+\beta),
$$

is positive.

In general, the sign of the expected utility premium will depend on the agent's level of wealth $w$. However, for agents whose preferences are characterized by a utility function exhibiting constant absolute risk aversion (CARA), the sign of the expected utility premium - and, hence, their preference ordering - depends only on their coefficient of risk aversion $a$. Indeed, because CARA preferences are (up to additive and positive multiplicative constants) uniquely represented by $u(w ; a)=$ $1-e^{-a w}$, the $\$ 1$ investment in $X$ is preferred by any CARA individual for which

$$
\mathbf{E}\left[e^{-a(X-\beta)}\right] \geq 1
$$

2.2. A Family of Riskiness Measures. For arbitrary $X \in \mathcal{G}$ and $\beta \geq 1$, define

$$
a^{*}(X, \beta)=\inf \left\{a>0: \mathbf{E}\left[e^{-a(X-\beta)}\right]>1\right\} .
$$

\footnotetext{
${ }^{4}$ Recall that the gross rate of return is computed as $P_{t} / P_{t-1}$, where $P_{t}$ denotes the price of the financial asset at time $t$.

${ }^{5}$ The actual amount invested is not important in our subsequent analysis since riskiness is measured per dollar invested.
} 
The parameter $a^{*}(X, \beta)$ is the maximum level of risk aversion at which a CARA agent is indifferent between the guaranteed rate of return $\beta$, and the risky investment in $X$.

Following Aumann and Serrano (2008), we define the riskiness (per dollar invested) of $X$ relative to $\beta$ as

$$
R(X, \beta) \equiv \lim _{a \rightarrow a^{*}(X, \beta)} \frac{1}{a} .
$$

The limiting operation is required to accommodate cases in which $a^{*}(X, \beta)=0$ or $a^{*}(X, \beta)=\infty$. In particular, when $\mathbf{P}[X>\beta]=1$, we have $a^{*}(X, \beta)=0$ since $X$ first-order stochastically dominates $\beta$ and, hence, every CARA agent would accept the risky investment strategy. When $\mathbf{P}[X<\beta]>0$ and $\mathbf{E}[X]<\beta$, no CARA agent would prefer the risky investment strategy to their fixed level of wealth and thus $a^{*}(X, \beta)=\infty$. Otherwise, when $\mathbf{P}[X<\beta]>0$ and $\mathbf{E}[X]>\beta$, the parameter $a^{*}(X, \beta)$ is a strictly positive real number .

To see that the riskiness ordering of two assets may change when the benchmark rate of return $\beta$ is varied even only slightly, consider Figure 1 in which we plot expected utility premiums (with $w$ normalized to zero) for weekly gross returns on the FTSE 100 and the CAC 40 stock indices. ${ }^{6}$ Here we see a reversal in the riskiness ordering of the two indices after only a $0.1 \%$ increase in the benchmark return. Indeed, panel (a) indicates that CAC 40 is riskier than the FTSE 100 relative to $\beta=1$, whereas panel (b) indicates that FTSE 100 is riskier than the CAC 40 index relative to $\beta=1.001$.

\footnotetext{
${ }^{6}$ These data are fully described in Section 5.
} 


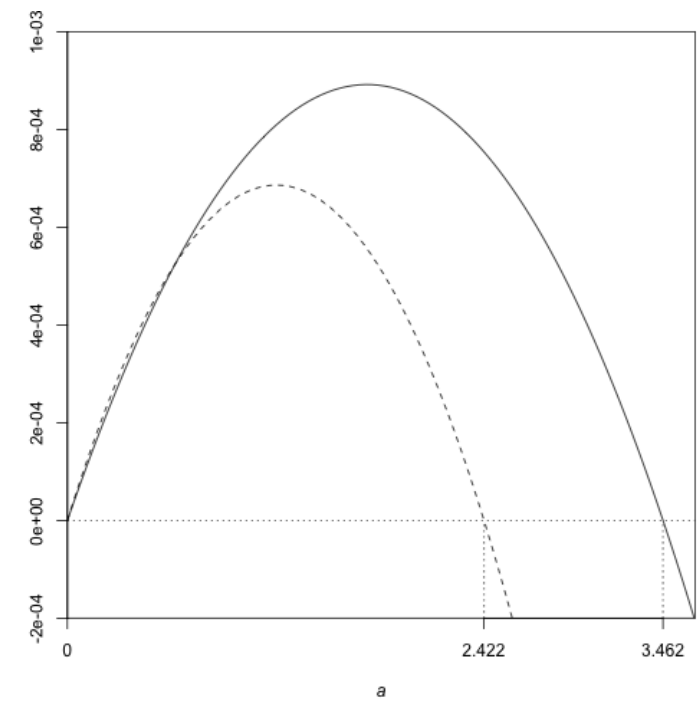

(a) $\beta=1$

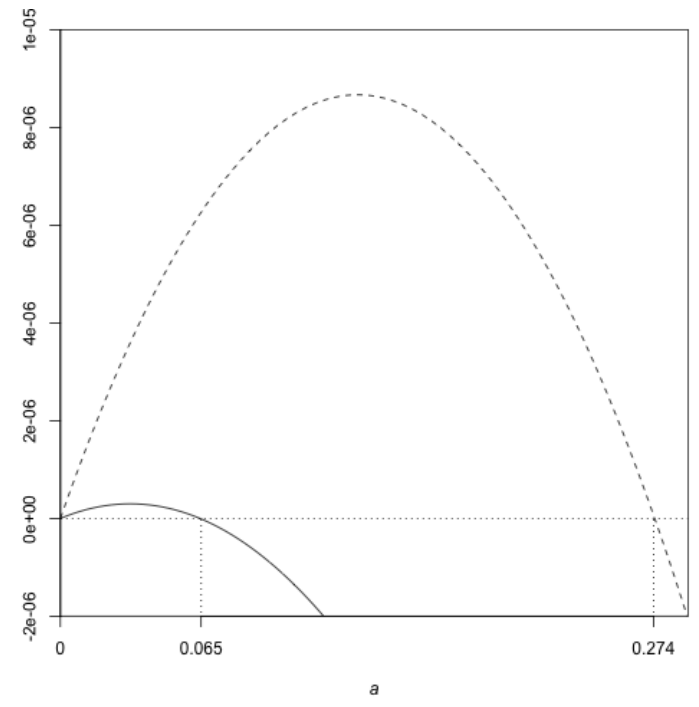

(b) $\beta=1.001$

FiguRE 1. Expected utility premiums for weekly gross returns on the FTSE 100 (solid line) and the CAC 40 (dashed line) indices.

Under certain conditions, the riskiness ordering of two assets may of course be invariant to an entire range of benchmark levels of return. The specific nature of these conditions for the range $\beta \geq 1$ is the content of Proposition 2.1 below: $^{7}$

Proposition 2.1. $R(X, \beta) \leq R(Y, \beta)$ for every $\beta \geq 1$ if and only if 8

$$
\mathbf{E}\left[e^{-a X}\right] \leq \mathbf{E}\left[e^{-a Y}\right] \quad \text { for every } a \in\left(0, a_{0}\right)
$$

where $a_{0} \equiv \inf \left\{a \in \mathbb{R}_{+}: \mathbf{E}\left[e^{-a(X-1)}\right]>1\right\}$.

Because $L_{X}(a) \equiv \mathbf{E}\left[e^{-a X}\right]$ is the Laplace transform associated with the random variable $X$, Proposition (2.1) says that the riskiness ordering is invariant to the ${ }^{7}$ Here we concentrate on the range $\beta \geq 1$ consisting of all benchmark levels of return that are positive. The modifications necessary for including benchmark levels of return $\beta$ in some interval $[\underline{R}, \bar{R}]$ other than $[1, \infty)$ are straightforward and left to the reader.

${ }^{8}$ Allow for the case that $a_{0}=+\infty$. 
choice of $\beta \geq 1$ whenever the respective Laplace transforms are ordered on the interval $\left[0, a_{0}\right]$.

We note here that the Laplace transform and its connection to choice under risk appears elsewhere in the literature. Whitmore (1989), for example, studies the (unrestricted) Laplace order and its connection to the ordering of certainty equivalent functions. Thistle (1993) also connects the Laplace order to the problem of choice under risk, showing that all expected utility maximizers with completely monotone von-Neumann-Morgenstern utility functions agree on their preference ordering of two risky assets whenever the assets are ordered by their Laplace transforms. See also Denuit (2001), Bennett (2008), and references therein for a review of the Laplace order and its application to problems in economics, finance, and actuarial science.

\section{INFERENCE}

In this section, we develop a statistical test that can be used to infer whether $R(X, \beta) \leq R(Y, \beta)$ holds over a range of benchmark levels of return. ${ }^{9}$ This work extends in various directions the paper by Knight and Satchell (2008), in which the authors test a set of necessary conditions for Laplace dominance. In particular, the procedure developed herein (i) allows for general forms of dependence (mutual and serial); (ii) allows for the range of dominance to be determined endogenously; and (iii) tests the full set of restrictions implied by (restricted) Laplace dominance.

3.1. Statistical Hypotheses. Our interest centers on constructing a statistical test that can be used to infer whether the riskiness ordering induced by $R(\cdot, \beta)$ is invariant to the choice of benchmark $\beta$. From Proposition 2.1, this amounts to a

\footnotetext{
$\overline{{ }^{9} \text { Inference for }} R(X, \beta)$ with $\beta>1$ fixed is treated in Homm and Pigorsch (2012).
} 
test of

$$
\mathrm{H}_{0}: \mathbf{E}\left[e^{-a X}\right] \leq \mathbf{E}\left[e^{-a Y}\right] \text { for every } a \in\left[0, a_{0}\right]
$$

against

$$
\mathrm{H}_{1}: \mathbf{E}\left[e^{-a X}\right]>\mathbf{E}\left[e^{-a Y}\right] \text { for some } a \in\left[0, a_{0}\right]
$$

where $a_{0} \equiv \inf \left\{a \in \mathbb{R}_{+}: \mathbf{E}\left[e^{-a(X-1)}\right]>1\right\}$.

This is a complex problem because we are dealing with curves, which are infinitedimensional parameters. To construct a test for $\mathrm{H}_{0}$ against $\mathrm{H}_{1}$, therefore, we introduce a one-dimensional parameter $\theta$ such that the hypotheses can be reformulated as

$$
\mathrm{H}_{0}: \theta=0 \text { against } \mathrm{H}_{1}: \theta>0
$$

To do this, we use the fact that

$$
\mathbf{E}\left[e^{-a X}\right] \leq \mathbf{E}\left[e^{-a Y}\right] \Leftrightarrow \int_{0}^{\infty} e^{-a t}(F(t)-G(t)) d t \leq 0
$$

to define $\theta$ as

$$
\theta \equiv \sup _{a \in\left[0, a_{0}\right]} \int_{0}^{\infty} e^{-a t}(F(t)-G(t)) d t
$$

Because the supremum is zero whenever $\mathrm{H}_{0}$ is true and is otherwise strictly greater than zero, this definition of $\theta$ indeed satisfies (6).

3.2. Estimator and Asymptotic Behavior. Let $\left\{\left(X_{i}, Y_{i}\right), 1 \leq i \leq n\right\}$ denote a sequence of $n$ observations. We assume that the marginal distributions $F$ and $G$ associated with $X$ and $Y$ are continuous with common support $[0, \infty)$ and with $\min \{\mathbf{E}[X], \mathbf{E}[Y]\}>1$. Additionally, we assume that the probability mechanism generating the data satisfies the following weak dependency condition. 
Assumption 3.1. $\left\{\left(X_{i}, Y_{i}\right), 1 \leq i \leq n\right\}$ is a strictly stationary and $\alpha$-mixing sequence such that $\alpha_{n}=O\left(n^{-\nu}\right), \nu>3$.

Estimation of the parameter $\theta$ as defined in equation (7) requires estimation of the unknown parameter $a_{0}$ together with the unknown distribution functions $F$ and $G$. To estimate $a_{0}$, we use

$$
a_{n} \equiv \underset{a>0}{\operatorname{argmax}} Q_{n}(a)
$$

where

$$
Q_{n}(a)=-\left[\frac{1}{n} \sum_{i=1}^{n}\left(e^{-a\left(X_{i}-1\right)}-1\right)\right]^{2}
$$

Proposition 3.1 below shows that $a_{n}$ is a consistent estimator of $a_{0}$ under our maintained assumptions.

Proposition 3.1. Suppose that Assumption 3.1 is satisfied, and that $\mathbf{E}[X]>1$ and $\mathbf{P}[X<1]=p>0$. Then, the estimator $a_{n}$ as defined in equation (8) satisfies $a_{n} \stackrel{\mathbf{P}}{\rightarrow} a_{0}$.

With the estimator $a_{n}$ of $a_{0}$ in hand, we take as our test statistic

$$
\sqrt{n} \hat{\theta}_{n}=\sup _{a \in\left[0, a_{n}\right]} \int_{0}^{\infty} e^{-a t} \sqrt{n}\left(F_{n}(t)-G_{n}(t)\right) d t
$$

where $F_{n}$ and $G_{n}$ are the empirical distribution functions based on the samples $X_{1}, X_{2}, \ldots, X_{n}$ and $Y_{1}, Y_{2}, \ldots, Y_{n}$.

To complete the description of our testing procedure, we need only provide a means for determining the appropriate threshold or critical value for rejection of the null hypothesis $\mathrm{H}_{0}$. Since it is large values of $\sqrt{n} \hat{\theta}_{n}$ that constitute evidence against $\mathrm{H}_{0}$, the task before us amounts to determining a (possibly data-dependent) 
threshold $c_{n}(\alpha)$ for a test at the nominal level $\alpha$ in such a way that

$$
\lim _{n \rightarrow \infty} \mathbf{P}\left[\sqrt{n} \hat{\theta}_{n}>c_{n}(\alpha) \mid \mathrm{H}_{0}\right] \leq \alpha
$$

with equality holding on the boundary of the null where $F=G$.

In the next subsection we outline a resampling procedure that gives a threshold $c_{n}(\alpha)$ satisfying (11). Note that we are forced to use resampling or other simulationbased methods here because the asymptotic distribution of $\sqrt{n} \hat{\theta}_{n}$ under the null, as shown in Theorem 3.1 below, depends on the (unknown) underlying distributions $F$ and $G$.

Theorem 3.1. Suppose that $F$ and $G$ are continuous and that Assumption 3.1 is satisfied. Then, on the boundary of the null hypothesis where $F=G$,

$$
\sqrt{n} \hat{\theta}_{n} \stackrel{W}{\rightarrow} \sup _{a \in\left[0, a_{0}\right]} \int_{0}^{\infty} e^{-a t} \mathbb{G}(t) d t
$$

where $\mathbb{G}(t)$ is a Gaussian process with covariance kernel as specified in equation (18) of the technical appendix.

3.3. Estimating Critical Values. Since the test statistic $\sqrt{n} \hat{\theta}_{n}$ is not asymptotically pivotal, we turn to the moving block bootstrap (Künsch 1989, Liu and Singh 1992) to compute the critical values for the test. To outline this procedure, let $\mathcal{B}_{i}=\left\{\left(X_{i}, Y_{i}\right) \ldots,\left(X_{i+l-1}, Y_{i+l-1}\right)\right\}$ represent a block of length $\ell$ formed from the sample $\left\{\left(X_{i}, Y_{i}\right), 1 \leq i \leq n\right\}$.

Bootstrap samples are generated by sampling $k$ blocks uniformly with replacement from the collection $\left\{\mathcal{B}_{i}, 1 \leq i \leq N\right\}$ where $N=n-\ell+1$ and $k=n / \ell .{ }^{10}$ The

\footnotetext{
${ }^{10}$ For ease of exposition, we assume that $n / \ell$ is integer-valued. When $n / \ell$ is not integer-valued, we may of course take $k=\lceil n / \ell\rceil$ so that $k$ is the smallest integer satisfying $k \ell \geq n$.
} 
bootstrap version of $\sqrt{n} \hat{\theta}_{n}$ is then defined by

$$
\sqrt{n} \hat{\theta}_{n}^{*}=\sup _{a \in\left[0, a_{n}\right]} \int_{0}^{\infty} e^{-a t} \sqrt{n}\left(F_{n}^{*}(t)-G_{n}^{*}(t)-\mathbf{E}_{*}\left[F_{n}^{*}(t)-G_{n}^{*}(t)\right]\right) d t
$$

where $F_{n}^{*}$ and $G_{n}^{*}$ denote the (marginal) empirical distributions based on the $n=k \ell$ bootstrap pseudo observations, and

$$
\mathbf{E}_{*}\left[F_{n}^{*}(t)-G_{n}^{*}(t)\right]=\frac{1}{N \ell} \sum_{i=1}^{N} \sum_{j=i}^{i+\ell-1}\left(1_{X_{j} \leq t}-1_{Y_{j} \leq t}\right)
$$

is a re-centering term, which is included so that the bootstrap process mimics the boundary case in which $F=G$.

The bootstrap estimate of the nominal $1-\alpha$ level critical value is then defined as

$$
c_{n}(\alpha)=\inf \left\{x: \mathbf{P}_{*}\left[\sqrt{n} \hat{\theta}_{n}^{*}>x\right] \leq \alpha\right\}
$$

where $\mathbf{P}_{*}$ denotes the conditional distribution of $\sqrt{n} \hat{\theta}_{n}^{*}$ given $\left(X_{1}, Y_{1}\right),\left(X_{2}, Y_{2}\right), \ldots,\left(X_{n}, Y_{n}\right)$.

Theorem (3.2) below shows that, under mild conditions, our proposed test with critical values determined from (15) is (i) asymptotically valid and (ii) consistent.

Theorem 3.2. Suppose that Assumption 3.1 is satisfied, and that $\ell \rightarrow \infty$ and $\ell=O\left(n^{\epsilon}\right)$ as $n \rightarrow \infty$ for $0<\epsilon<\frac{1}{3}$. Then:

i. If $H_{0}$ is true, then for $\alpha<1 / 2$

$$
\lim _{n \rightarrow \infty} \mathbf{P}\left[\sqrt{n} \hat{\theta}_{n}>c_{n}(\alpha)\right] \leq \alpha
$$

with equality holding on the boundary of the null hypotheses where $F=G$. ii. If $H_{0}$ is false, then

$$
\lim _{n \rightarrow \infty} \mathbf{P}\left[\sqrt{n} \hat{\theta}_{n}>c_{n}(\alpha)\right]=1
$$




\section{Simulations}

In this section, we examine the finite-sample performance of our proposed test procedure by way of several Monte Carlo experiments. To do this, we consider various specifications of the bivariate process

$$
X_{k, i}=\lambda X_{k, i-1}+W_{k, i} 1_{U_{k, i}=1}+Z_{k, i} 1_{U_{k, i}=2}, \quad k=1,2, \quad i=1, \ldots, n,
$$

where $W_{k, i}, Z_{k, i}$, and $U_{k, i}$ are i.i.d. processes with $W_{k} \sim \operatorname{Gamma}\left(1, \theta_{k}\right), Z_{k} \sim$ $\operatorname{Gamma}\left(2, \theta_{k}\right)$, and $U_{k} \sim \operatorname{Binomial}\left(\gamma_{k}, 1-\lambda\right)$ for $\gamma_{k} \in\{1,2\}$.

This process is particularly attractive because it yields simple closed forms for the Laplace transforms associated with $X_{1}$ and $X_{2}$. Indeed, using a result from Gaver and Lewis (1980), we have $X_{k} \sim \operatorname{Gamma}\left(\gamma_{k}, \theta_{k}\right)$, which implies that the Laplace transform for $X_{k}$ is $\mathbf{E}\left[e^{-a X_{k}}\right]=\left(1+\theta_{k} a\right)^{-\gamma_{k}}$. Additionally, this process allows us to easily control the degree of serial dependence in $X_{k}(k=1,2)$ through the parameter $\lambda$ and also the degree of dependence between $X_{1}$ and $X_{2}$ by manipulating the dependence between the pairs $\left(W_{1, i}, W_{2, i}\right)$ and $\left(Z_{1, i}, Z_{2, i}\right)$.

All of our experimental designs are derived from the following two base cases:

Case I: $\gamma_{1}=\gamma_{2}=2$ and $\theta_{1}=\theta_{2}=1$.

Case II: $\gamma_{1}=2, \gamma_{2}=1, \theta_{1}=1$, and $\theta_{2}=3$.

In Case I, the null hypothesis $\mathrm{H}_{0}: \mathbf{E}\left[e^{-a X_{1}}\right] \leq \mathbf{E}\left[e^{-a X_{2}}\right]$ for every $a \in\left[0, a_{0}\right]$ is true, ${ }^{11}$ whereas in Case II, this same null hypothesis is false (indeed, the Laplace transforms for $X_{1}$ and $X_{2}$ intersect at $a=1<a_{0}$; see Figure 2).

We consider situations with $(\lambda=0.1)$ and without $(\lambda=0)$ serial dependence. Also, we generate the pair $W_{1}$ and $W_{2}$ using a bivariate Gaussian copula with correlation parameter equal to $\rho_{W}$, and the pair $Z_{1}$ and $Z_{2}$ using a bivariate Gaussian copula

\footnotetext{
$\overline{{ }^{11} \text { The value of }} a_{0}$ in both cases is approximately 2.5
} 
with correlation parameter equal to $\rho_{Z}=\rho_{W}$. Under this setup, the correlation between $X_{1}$ and $X_{2}$ can be computed as

$$
\rho_{X} \equiv \operatorname{Corr}\left(X_{1}, X_{2}\right)=\frac{p_{1} p_{2} \rho_{W}+2 q_{1} q_{2} \rho_{Z}}{\sqrt{\gamma_{1} \gamma_{2}}\left(1-\lambda^{2}\right)}
$$

where $p_{k} \equiv \mathbf{P}\left[U_{k}=1\right]=\gamma_{k}(1-\lambda) \lambda^{\gamma_{k}-1}$ and $q_{k} \equiv \mathbf{P}\left[U_{k}=2\right]=\left(\gamma_{k}-1\right)(1-$ $\lambda)^{2} \lambda^{\gamma_{k}-2}$. Clearly, for $\lambda=0$ (no serial dependence), the level of cross-correlation $\rho_{X}$ is always zero. However, for $\lambda=0.1$ (positive serial dependence), we choose values of $\rho_{W}$ (recall that $\rho_{Z}=\rho_{W}$ ) so as to generate levels of cross-correlation $\rho_{X} \in\{0,0.1,0.2,0.4,0.6\}$ for Case I and $\rho_{X} \in\{0,0.1\}$ for Case II. ${ }^{12}$

Rejection rates at the $5 \%$ nominal level for Cases I (null) and II (alternative) are given in Tables 1 and 2, respectively. For each case, we consider sample sizes $n=50$ and $n=500$, with block size $\ell \in\{1,2,3,4\}$ for $n=50$, and $\ell \in\{1,3, \ldots, 13\}$ for $n=500$. However, when the serial dependence parameter $\lambda$ is zero, we use only $\ell=1$. These results are based on 10,000 replications with $B=499$ bootstrap replications.

The results in Table 1 show that the empirical size of the proposed test tends to be close to the nominal $5 \%$ level for both $n=50$ and $n=500$ in the absence of serial dependence. When there is serial dependence, the test tends to over-reject at both sample sizes with the degree of over-rejection tending to be largest when when the block length is chosen too small. In particular, the size distortion is relatively large for sample sizes as great as $n=500$ when there is serial dependence and the block size is misspecified at $\ell=1$ (that is, when serial dependence is present but ignored).

\footnotetext{
${ }^{12}$ Note that, even with $\rho_{W}=\rho_{Z}=1$, we have $\rho_{X}=0.68$ in Case I and $\rho_{X}=0.12$ in Case II.
} 
Figure 2. Difference between Laplace transforms for $X_{1}$ and $X_{2}$ in Case II.

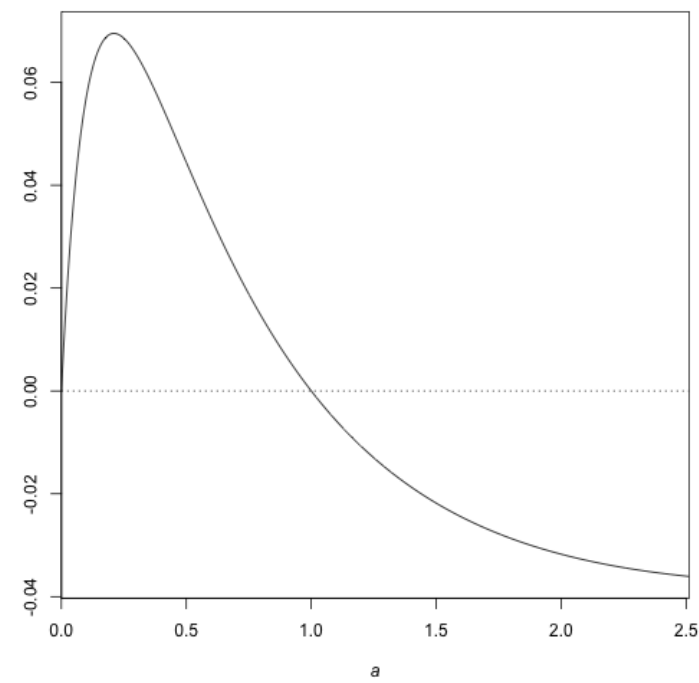

TABle 1. Empirical Rejection Probabilities (Case I: Null).

\begin{tabular}{lccccccc}
\hline & & \multicolumn{7}{c}{$\lambda=0.1$} \\
\cline { 3 - 7 }$n$ & $\ell$ & $\lambda=0$ & $\rho_{X}=0$ & $\rho_{X}=0.1$ & $\rho_{X}=0.2$ & $\rho_{X}=0.4$ & $\rho_{X}=0.6$ \\
\hline 50 & 1 & 0.054 & 0.078 & 0.074 & 0.069 & 0.074 & 0.073 \\
& 2 & - & 0.069 & 0.068 & 0.064 & 0.069 & 0.066 \\
& 3 & - & 0.070 & 0.067 & 0.065 & 0.067 & 0.065 \\
& 4 & - & 0.071 & 0.068 & 0.067 & 0.069 & 0.068 \\
500 & 1 & 0.049 & 0.073 & 0.076 & 0.074 & 0.068 & 0.069 \\
& 3 & - & 0.060 & 0.061 & 0.060 & 0.054 & 0.058 \\
& 5 & - & 0.060 & 0.058 & 0.058 & 0.052 & 0.056 \\
& 7 & - & 0.058 & 0.058 & 0.058 & 0.051 & 0.056 \\
& 9 & - & 0.060 & 0.058 & 0.059 & 0.053 & 0.055 \\
& 11 & - & 0.059 & 0.057 & 0.058 & 0.054 & 0.057 \\
& 13 & - & 0.059 & 0.060 & 0.060 & 0.054 & 0.056
\end{tabular}

Notes: For $\lambda=0$ (no serial dependence), the level of cross-correlation $\rho_{X}$ is always zero.

While the empirical size of the test does not seem to be affected by the level of cross-correlation, Table 2 shows that the empirical power of the proposed test tends to increase with the level of both serial- and cross-correlation. More generally, we see 
TABle 2. Empirical Rejection Probabilities (Case II: Alternatives)

\begin{tabular}{lcccc}
\hline & & & \multicolumn{2}{c}{$\lambda=0.1$} \\
\cline { 4 - 5 }$n$ & $\ell$ & $\lambda=0$ & $\rho_{X}=0$ & $\rho_{X}=0.1$ \\
\hline 50 & 1 & 0.336 & 0.354 & 0.386 \\
& 2 & - & 0.333 & 0.361 \\
& 3 & - & 0.325 & 0.355 \\
& 4 & - & 0.329 & 0.356 \\
500 & 1 & 0.997 & 0.998 & 0.999 \\
& 3 & - & 0.995 & 0.997 \\
& 5 & - & 0.994 & 0.997 \\
& 7 & - & 0.993 & 0.997 \\
& 9 & - & 0.993 & 0.996 \\
& 11 & - & 0.993 & 0.996 \\
& 13 & - & 0.993 & 0.996 \\
\hline
\end{tabular}

Notes: For $\lambda=0$ (no serial dependence), the level of cross-correlation $\rho_{X}$ is always zero.

that the empirical power of the test increases towards one when the sample size is increased, demonstrating the consistency of the test (as was established in Theorem $3.2)$.

\section{Empirical Illustration}

In this section, we use the proposed test to examine the riskiness of three major European stock indices: the CAC 40 (Paris), the DAX (Frankfurt), and the FTSE 100 (London). The data, which was obtained from Yahoo! Finance, consists of $n=1042$ weekly gross returns covering the period 18 January, 1993 - 31 December, 2012.

Descriptive statistics on the weekly gross returns are provided in Table 3. For each series, the minimum observation is below one, while the mean is above one, thereby satisfying the conditions in Proposition 3.1. Moreover, each series has a low level of first-order autocorrelation (less than 0.1 in absolute value), which is 
statistically significant only in the case of the FTSE 100 and the CAC 40. Table 3 also presents Augmented Dickey-Fuller (ADF) test statistics for each series, all of which are statistically significant at the $1 \%$ level, leading us to reject the null of non-stationarity. Not shown in Table 3 are the levels of cross-correlation, which range between 0.807 (for the DAX and the FTSE 100) and 0.867 (for the DAX and the CAC 40).

TABLE 3. Descriptive statistics

\begin{tabular}{rllllll}
\hline & & & & \multicolumn{3}{l}{ First-order } \\
& Min. & Max. & Mean & StdDev. & Autocorr. & ADF \\
\hline FTSE 100 & 0.790 & 1.134 & 1.001 & 0.024 & $-0.086(0.031)$ & -10.888 \\
DAX & 0.784 & 1.161 & 1.002 & 0.032 & $-0.044(0.031)$ & -9.560 \\
CAC 40 & 0.778 & 1.132 & 1.001 & 0.030 & $-0.087(0.031)$ & -9.842 \\
\hline
\end{tabular}

Notes: Standard errors for the estimates of first-order autocorrelation are given in brackets.

In Table 4 , we report intervals of $p$-values $\left[p_{\min }, p_{\max }\right]$ computed over the range of bootstrap block lengths $\ell \in\{1, \ldots, 20\}$ (here, we use $B=1,999$ bootstrap replications). Given these results, we reject the null of the CAC 40 dominating the DAX. We are also able to reject, albeit at a slightly higher level $\alpha$, the null of the FTSE 100 dominating the DAX.

TABLE $4 . a_{0}$ estimates together with $\left[p_{\min }, p_{\max }\right]$ for dominance tests

\begin{tabular}{rcccc}
\hline & $a_{n}$ & FTSE 100 & DAX & CAC 40 \\
\hline FTSE 100 & 3.462 & - & {$[0.067,0.092]$} & {$[0.450,0.495]$} \\
DAX & 3.871 & {$[1,1]$} & - & {$[1,1]$} \\
CAC 40 & 2.422 & {$[0.340,0.375]$} & {$[0.008,0.047]$} & - \\
\hline
\end{tabular}

On the other hand, we fail to reject the null of the DAX dominating the FTSE 100 and the DAX dominating the CAC 40 (the test statistics are zero in each case). We also fail to reject the null of the FTSE 100 dominating the CAC 40 and vice-versa. 
Taken together, these results suggest that the CAC 40 and the FTSE 100 are both riskier (relative to any benchmark $\beta \geq 1$ ) than the DAX. In other words, the CAC 40 and the FTSE 100 are both found to be uniformly riskier than the DAX.

In Figure 3, we plot $R\left(X_{k}, \beta\right)$, for $k \in\{1,2,3\}$, as a function of $\beta$. Note that, for $1 \leq \beta \leq \max \left\{\max \left(X_{1}\right), \max \left(X_{2}\right), \max \left(X_{3}\right)\right\}=1.002$, the level of riskiness is lower for the DAX than for either the FTSE 100 or the CAC 40 (recall that, for $\beta>\max \left(X_{k}\right)$, we have $\left.R\left(X_{k}, \beta\right)=\infty\right)$. This is consistent with the fact that our test fails to reject the null of the DAX dominating either the FTSE 100 or the CAC 40, but does reject the null of either the FTSE 100 or the CAC 40 dominating the DAX.

Figure 3. The riskiness measure $R\left(X_{k}, \beta\right)$ as a function of $\beta$ for returns on three stock indices

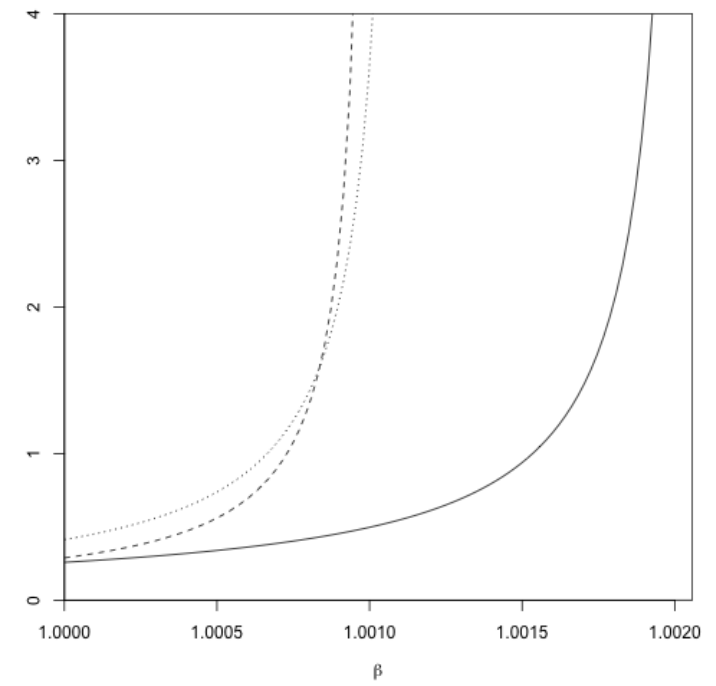

Notes: The solid line corresponds to the DAX, the dashed line corresponds to the FTSE 100, and the dotted line corresponds to the CAC 40.

Figure 3 also shows that the level of riskiness for the FTSE 100 is initially lower than that for the $\mathrm{CAC} 40$, but becomes greater at $\beta \approx 1.0008$, which is consistent with the fact that our test fails to reject the null of the FTSE 100 dominating the 
CAC 40 and vice-versa (see also Figure 4a, in which we plot the difference in the Laplace transforms for the FTSE 100 and the CAC 40). That one cannot obtain a uniform ordering may also be regarded as informative, therefore, in that it alerts users of the A-S measure to a possible reversal in the riskiness ordering when the benchmark is varied.

FiguRE 4. Difference between Laplace transforms for returns on two pairs of stock indices

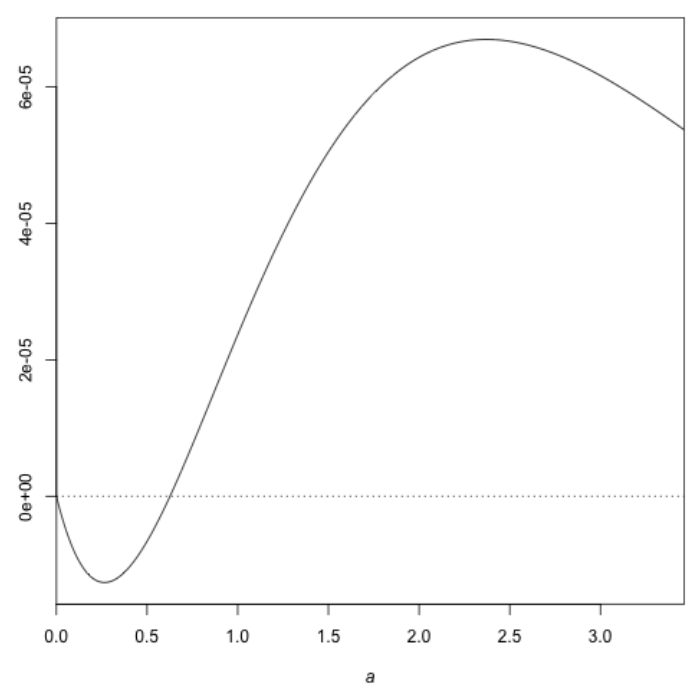

(a) FTSE 100 vs. CAC 40

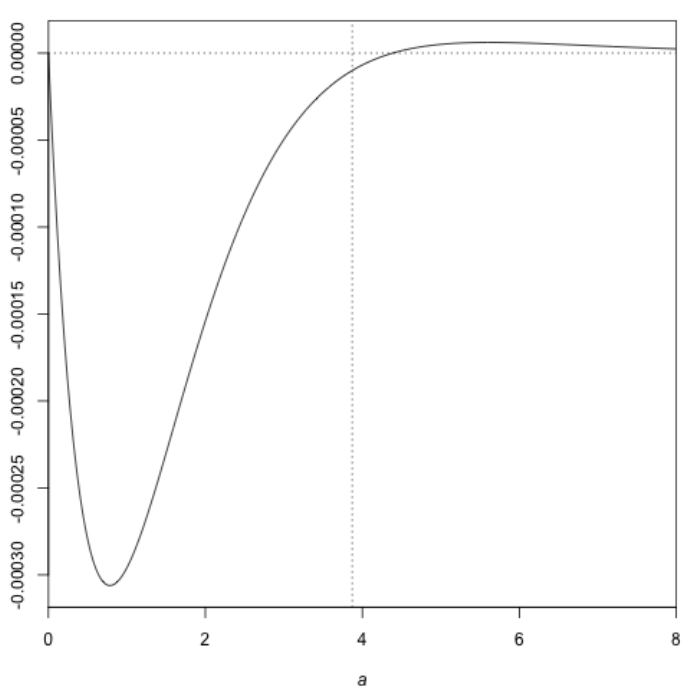

(b) DAX vs. FTSE 100

Notes: In (a), the upper limit of the horizontal axis is $a_{n}=3.462$ (for the FTSE 100). The dotted vertical line in (b) corresponds to $a_{n}=3.871$ (for the DAX).

We conclude this section with a closer look at the role of the interval $\left[0, a_{0}\right]$ in our test of (restricted) Laplace dominance. In Figure 4b, for example, we plot the difference in Laplace transforms for the DAX and the FTSE 100. As is clear from this figure, the Laplace transform for the DAX is everywhere below the Laplace transform for the FTSE up to $a_{n}=3.871$ (the upper threshold for the DAX), which accounts for the fact that the test statistic for this comparison is equal to zero. Interestingly, however, the Laplace transform for the DAX is actually above the 
Laplace form for the the FTSE 100 for some $a>a_{n}$. In other words, the DAX does not Laplace dominate the FTSE 100 over the entire real line (at least not in-sample), but it is does appear to dominate the FTSE 100 in the restricted case (i.e., up to $\left.a_{n}\right)$, which is all that is necessary when our objective is to infer a riskiness ordering that is uniform over $\beta \geq 1$.

\section{Concluding Remarks}

The riskiness index of Aumann and Serrano (2008) was constructed by comparing individual risky assets to a common (subjective) benchmark yielding a gross return of one. In this paper, we have generalized the riskiness index of Aumann and Serrano (2008) to allow for benchmarks that have positive net returns and corresponding levels of gross return greater than one. While the riskiness ordering can, in some cases, be highly sensitive to the choice of benchmark, we have established that the riskiness ordering of a given pair of assets is invariant to the choice of measure within this generalized class whenever one asset dominates the other in a restricted version of the Laplace order. To make this result useful in practical settings where only samples from the underlying distributions are observed, we have also developed a statistical test of this dominance condition and demonstrated its usefulness by way of several simulation experiments and an empirical application. 


\section{Appendix A. Appendix}

\section{A.1. Properties of the Measures.}

Proof of Proposition 2.1. Suppose that $\mathbf{E}\left[e^{-a X}\right] \leq \mathbf{E}\left[e^{-a Y}\right]$ for every $a \in\left[0, a_{0}\right]$. Then,

$$
1=\mathbf{E}\left[e^{-a^{*}(X, \beta)(X-\beta)}\right] \leq \mathbf{E}\left[e^{-a^{*}(X, \beta)(Y-\beta)}\right]
$$

for every $\beta \geq 1$ and, hence, $a^{*}(X, \beta) \geq a^{*}(Y, \beta)$ for every $\beta \geq 1$. This completes the proof of the "if" part. To prove the "only if" part, suppose that $R(X, \beta) \leq R(Y, \beta)$ for every $\beta \geq 1$, in which case $a^{*}(X, \beta) \geq a^{*}(Y, \beta)$ for every $\beta \geq 1$. The desired result then follows since

$$
\mathbf{E}\left[e^{-a^{*}(X, \beta)(X-\beta)}\right] \leq \mathbf{E}\left[e^{-a^{*}(X, 1)(Y-\beta)}\right] \leq \mathbf{E}\left[e^{-a^{*}(Y, \beta)(Y-\beta)}\right]
$$

holds for every $\beta \geq 1$.

\section{A.2. Large sample theory. Define}

$$
\Delta_{n}(t)=\left(F_{n}(t)-F(t)\right)-\left(G_{n}(t)-G(t)\right)
$$

We will make considerable use of the following classical result concerning the weak convergence of the scaled and centered empirical process $\sqrt{n} \Delta_{n}(t)$, formulated here as a lemma for convenient referencing:

Lemma A.1. Suppose that Assumption 3.1 is satisfied. Then,

$$
\sqrt{n} \Delta_{n}(t) \stackrel{W}{\rightarrow} \mathbb{G}(t)
$$


where $\mathbb{G}(t)$ is a Gaussian process with covariance kernel

$$
\begin{aligned}
\Gamma(s, t)= & F(s)(1-F(t))+\lim _{n \rightarrow \infty} \sum_{i \neq j}^{n}\left(\mathbf{E}\left[1_{X_{i} \leq s} 1_{X_{j} \leq t}\right]-F(s) F(t)\right) \\
& +G(s)(1-G(t))+\lim _{n \rightarrow \infty} \sum_{i \neq j}^{n}\left(\mathbf{E}\left[1_{Y_{i} \leq s} 1_{Y_{j} \leq t}\right]-G(s) G(t)\right) \\
& +\lim _{n \rightarrow \infty} \sum_{i}^{n} \sum_{j}^{n}\left(\mathbf{E}\left[1_{X_{i} \leq s} 1_{Y_{j} \leq t}\right]-F(s) G(t)\right) \\
& +\lim _{n \rightarrow \infty} \sum_{i}^{n} \sum_{j}^{n}\left(\mathbf{E}\left[1_{X_{i} \leq t} 1_{Y_{j} \leq s}\right]-F(t) G(s)\right)
\end{aligned}
$$

For additional background on weak convergence results for empirical processes with dependent data, we refer the reader to Radulović (2002) and references therein.

Proof of Proposition 3.1. Define the function $Q_{0}: \mathbf{R}_{++} \rightarrow \mathbf{R}$ by

$$
Q_{0}(a)=-\left[\mathbf{E} e^{-a(X-1)}-1\right]^{2}
$$

The proof involves verifying the sufficient conditions for weak consistency as formulated in Theorem 2.7 of Newey and McFadden (1986). First, the conditions $\mathbf{E}[X]>1$ and $\mathbf{P}[X<1]$ imply that $Q_{0}(a)$ is strictly concave and uniquely maximized at $a_{0}>0$. Second, since $X_{i}$ is stationary and $\alpha$-mixing, so too is $Z_{i}=\exp \left(-a X_{i}-1\right)$. It therefore follows from the WLLN for ergodic stationary sequences that $n^{-1} \sum_{i=1}^{n} Z_{i} \stackrel{\mathbf{P}}{\rightarrow}$ $\mathbf{E}\left(Z_{i}\right)$ for all $a \in \mathbf{R}_{++}$, and hence that $Q_{n}(a) \stackrel{\mathbf{P}}{\rightarrow} Q_{0}(a)$ for all $a \in \mathbf{R}_{++}$.

Proof of Theorem 3.1. Define

$$
\Delta_{n}(t)=\left(F_{n}(t)-F(t)\right)-\left(G_{n}(t)-G(t)\right)
$$


so that $\sqrt{n} \hat{\theta}_{n}\left(a_{n}\right)$ can be equivalently rewritten as

$$
\sqrt{n} \hat{\theta}_{n}\left(a_{n}\right)=\sup _{a \in\left[0, a_{n}\right]}\left\{\int_{0}^{\infty} e^{-a t} \sqrt{n} \Delta_{n}(t) d t+\sqrt{n} \int_{0}^{\infty} e^{-a t}(F(t)-G(t)) d t\right\} .
$$

Since our objective is to establish the limiting distribution on the boundary of the null hypothesis where $F=G$, we may use the fact that the second integral is zero in this case to further simplify (21) as

$$
\sqrt{n} \hat{\theta}_{n}\left(a_{n}\right)=\sup _{a \in\left[0, a_{0}\right]} \int_{0}^{\infty} e^{-a t} \sqrt{n} \Delta_{n}(t) d t+\sqrt{n}\left[\hat{\theta}_{n}\left(a_{n}\right)-\hat{\theta}_{n}\left(a_{0}\right)\right] .
$$

The second term is asymptotically negligible and hence inconsequential to the limiting distribution. To see this, first note that

$$
\begin{aligned}
& \mathbf{P}\left[\sqrt{n}\left[\hat{\theta}_{n}\left(a_{n}\right)-\hat{\theta}_{n}\left(a_{0}\right)\right]>\epsilon\right] \\
& \quad \leq \mathbf{P}\left[\left|a_{n}-a_{0}\right| \sup _{a:\left|a-a_{0}\right|<\delta} \int_{0}^{\infty}-t e^{-a t} \sqrt{n} \Delta_{n}(t) d t>\epsilon\right]+\mathbf{P}\left[\left|a_{n}-a_{0}\right|>\delta\right],
\end{aligned}
$$

where $\delta$ is an arbitrary positive constant satisfying $a_{0}-\delta>0$. The second term in (23) vanishes as $n \rightarrow \infty$ since $\left|a_{n}-a_{0}\right|=o_{\mathbf{P}}(1)$ (**ref to earlier results**). The fact that $\left|a_{n}-a_{0}\right|=o_{\mathbf{P}}(1)$ further implies that the first term in (23) also vanishes as $n \rightarrow \infty$ provided that

$$
\sup _{a:\left|a-a_{0}\right|<\delta} \int_{0}^{\infty}-t e^{-a t} \sqrt{n} \Delta_{n}(t) d t=O_{\mathbf{P}}(1)
$$

Concentrating on the first term on the right-hand-side, we that note that assumption (3.1) is sufficient for Yoshihara's (1979) weak limit theorem, which, together with the classical Kolmogorov-Smirnov theorem, implies that

$$
\sup _{a \in\left[0, a_{0}\right]} \int_{0}^{\infty} e^{-a t} \sqrt{n} \Delta_{n}(t) d t \stackrel{W}{\rightarrow} \sup _{a \in\left[0, a_{0}\right]} \int_{0}^{\infty} e^{-a t} \mathbb{G}(t) d t
$$


where $\mathbb{G}(t)$ is a Gaussian process with covariance kernel (18).

The second term is inconsequential to the limiting distribution since it is asymptotically negligible. To verify this, fix $\delta$ such that $a_{0}-\delta>0$. Since

$$
\begin{aligned}
& \mathbf{P}\left[\sqrt{n}\left[\hat{\theta}_{n}\left(a_{n}\right)-\hat{\theta}_{n}\left(a_{0}\right)\right]>\epsilon\right] \\
& \quad \leq \mathbf{P}\left[\left|a_{n}-a_{0}\right| \sup _{a \in B_{\delta}\left(a_{0}\right)} \int_{0}^{\infty}-t e^{-a t} \sqrt{n} \Delta_{n}(t) d t>\epsilon\right]+\mathbf{P}\left[\left|a_{n}-a_{0}\right|>\delta\right] \\
& =o_{P}(1)
\end{aligned}
$$

we have

To characterize the limiting distribution $\sqrt{n} \hat{\theta}_{n}\left(a_{n}\right)$, we proceed in two steps. First, we will show that the second term in $(22)$ is $o_{P}(1)$. Since this result implies that the asymptotic distribution of $\sqrt{n} \hat{\theta}_{n}\left(a_{n}\right)$ is completely characterized by the asymptotic distribution of $\sqrt{n} \hat{\theta}_{n}\left(a_{0}\right)$, we will then turn our attention to characterizing the limiting distribution of $\sqrt{n} \hat{\theta}_{n}\left(a_{0}\right)$.

Let $\epsilon>0$ be given and pick $\delta>0$ such that $a_{0}-\delta>0$. Then,

$$
\begin{aligned}
& \mathbf{P}\left[\sqrt{n}\left[\hat{\theta}_{n}\left(a_{n}\right)-\hat{\theta}_{n}\left(a_{0}\right)\right]>\epsilon\right] \\
& \quad \leq \mathbf{P}\left[\left|a_{n}-a_{0}\right| \sup _{a \in B_{\delta}\left(a_{0}\right)} \int_{0}^{\infty}-t e^{-a t} \sqrt{n} \Delta_{n}(t) d t>\epsilon\right]+\mathbf{P}\left[\left|a_{n}-a_{0}\right|>\delta\right] \\
& =o_{P}(1)
\end{aligned}
$$

From here, we proceed in two steps. First, we establish the limiting distribution of the first term. We then show that the second

. Focusing on the integrand in (21), we have

$$
e^{-a t}\left(\sqrt{n} \Delta_{n}(t) \mathbf{1}_{\left[0, a_{0}\right]}+\sqrt{n} \Delta_{n}(t)\left(\mathbf{1}_{\left[0, a_{n}\right]}-\mathbf{1}_{\left[0, a_{0}\right]}\right)\right) \stackrel{W}{\rightarrow} e^{-a t} \mathbb{G}(t) \mathbf{1}_{\left[0, a_{0}\right]}
$$


where $\mathbb{G}(t)$ is a Gaussian process with covariance kernel (18). This is because

$$
\mathbf{1}_{\left[0, a_{n}\right]}-\mathbf{1}_{\left[0, a_{0}\right]}=o_{\mathbf{P}}(1)
$$

uniformly in $a \in \mathbf{R}_{+}$, and because Assumption (3.1) is sufficient for Yoshihara's (1979) weak limit theorem, which implies that

$$
\sqrt{n} \Delta_{n}(t)_{t \in \mathbf{R}_{+}} \stackrel{W}{\rightarrow} \mathbb{G}(t)_{t \in \mathbf{R}_{+}}
$$

The final step is an appeal to the continuous mapping theorem that gives

$$
\sqrt{n} \hat{\theta}_{n} \stackrel{W}{\rightarrow} \sup _{a \in\left[0, a_{0}\right]} \int_{0}^{\infty} e^{-a t} \mathbb{G}(t) d t
$$

thereby establishing (12) as the correct expression for the limiting distribution of $\sqrt{n} \hat{\theta}_{n}$

Proof of Theorem 3.2. The suppositions of the Theorem 3.2 are sufficient for the bootstrap CLT of Radulović (2002), which establishes that

$$
\sqrt{n}\left(F_{n}^{*}(t)-G_{n}^{*}(t)-\mathbf{E}_{*}\left[F_{n}^{*}(t)-G_{n}^{*}(t)\right]\right)_{t \in \mathbf{R}_{+}} \stackrel{W}{\rightarrow} \mathbb{G}(t)_{t \in \mathbf{R}_{+}}
$$

in probability, where $\mathbb{G}(t)$ is the Gaussian process of Theorem (3.1). Mimicking the proof of Theorem 3.1 thus gives

$$
\sqrt{n} \hat{\theta}_{n}^{*} \stackrel{W}{\rightarrow} \sup _{a \in\left[0, a_{0}\right]} \int_{0}^{\infty} e^{-a t} \mathbb{G}(t) d t
$$

in probability.

To establish part (i), it suffices to show that

$$
\lim _{n \rightarrow \infty} \mathbf{P}\left[\sqrt{n} \hat{\theta}_{n}>c_{n}(\alpha) \mid F=G\right]=\alpha
$$


for all $0<\alpha<1 / 2$. In light of (12) and (31), however, Theorem 1 of Beran (1984) implies that (32) is indeed satisfied if the distribution of $\sup _{a \in\left[0, a_{0}\right]} \int_{0}^{\infty} e^{-a t} \mathbb{G}(t) d t$ is continuous at its $1-\alpha$ quantile for all $0<\alpha<1 / 2$. To verify the latter, note that $\sup _{a \in\left[0, a_{0}\right]} \int_{0}^{\infty} e^{-a t} \mathbb{G}(t) d t$ is a convex functional of $\mathbb{G}(t)$ and, hence, from Theorem 11.1 of Davydov, Lifshits and Smorodina (1998) it follows that the distribution of $\sup _{a \in\left[0, a_{0}\right]} \int_{0}^{\infty} e^{-a t} \mathbb{G}(t) d t$ is continuous everywhere except possibly at zero. That the distribution of $\sup _{a \in\left[0, a_{0}\right]} \int_{0}^{\infty} e^{-a t} \mathbb{G}(t) d t$ is continuous at its $1-\alpha$ quantile for all $0<\alpha<1 / 2$ then follows as a direct consequence of the fact that

$$
\mathbf{P}\left[\sup _{a \in\left[0, a_{0}\right]} \int_{0}^{\infty} e^{-a t} \mathbb{G}(t) d t \leq 0\right] \leq \mathbf{P}[\inf \{t>0: \mathbb{G}(t)<0\}=0] \leq 1 / 2 .
$$

As for part (ii), note that

$$
\begin{aligned}
& \lim _{n \rightarrow \infty} \mathbf{P}\left[\sqrt{n} \hat{\theta}_{n}>c_{n}(\alpha)\right] \\
& \geq \lim _{n \rightarrow \infty} \mathbf{P}\left[\sup _{a \in\left[0, a_{n}\right]} \int_{0}^{\infty} e^{-a t} \sqrt{n} \Delta_{n}(t) d t>\sup _{a \in\left[0, a_{n}\right]} \int_{0}^{\infty} e^{-a t} \sqrt{n}(F(t)-G(t)) d t+c_{n}(\alpha)\right]
\end{aligned}
$$

Next, we use the fact that

$$
\sup _{a \in\left[0, a_{n}\right]} \int_{0}^{\infty} e^{-a t} \sqrt{n} \Delta_{n}(t) d t=O_{\mathbf{P}}(1)
$$

(c.f. Theorem 3.1), together with

$$
\sup _{a \in\left[0, a_{n}\right]} \int_{0}^{\infty} e^{-a t} \sqrt{n}(F(t)-G(t)) d t+c_{n}(\alpha) \stackrel{\mathbf{P}}{\rightarrow}-\infty
$$

which is implied by the continuity of $F$ and $G$ together with fact that $F(t)<G(t)$ for some $t \in\left[0, a_{0}\right]$ under $\mathrm{H}_{1}$, to obtain the desired result, namely that

$$
\lim _{n \rightarrow \infty} \mathbf{P}\left[\sqrt{n} \hat{\theta}_{n}>c_{n}(\alpha)\right] \rightarrow 1
$$


under $\mathrm{H}_{1}$. 


\section{REFERENCES}

Anderson, G. (1996), 'Nonparametric tests of stochastic dominance in income distributions', Econometrica 64(5), 1183-1193.

Aumann, R. and Serrano, R. (2008), 'An economic index of riskiness', Journal of Political Economy 116(5), 810-836.

Barrett, G. F. and Donald, S. G. (2003), 'Consistent tests for stochastic dominance', Econometrica 71(1), 71-104.

Bennett, C. (2008), 'Essays on estimation risk and stochastic dominance in economics'. Ph.D. Dissertation, University of Western Ontario.

Bennett, C. J. (2013), 'Inference for dominance relations', International Economic Review 54(4), 1309-1328.

Beran, R. J. (1984), 'Bootstrap methods in statistics', Jahresbericht der Deutschen MathematikerVereinigung 86, 14-30.

Davidson, R. and Duclos, J.-Y. (2000), 'Statistical inference for stochastic dominance and for the measurement of poverty and inequality', Econometrica 68(6), 1435-1464.

Davydov, I., Lifshits, M. and Smorodina, N. (1998), Local Properties of Distributions of Stochastic Functionals, American Mathematical Society, Providence, RI.

Denuit, M. (2001), 'Laplace transform ordering of actuarial quantities', Insurance: Mathematics and Economics 29(1), 83 - 102.

Gaver, D. P. and Lewis, P. A. W. (1980), 'First-order autoregressive gamma sequences and point processes', Advances in Applied Probability 12(3), 727-745.

Homm, U. and Pigorsch, C. (2012), 'Beyond the Sharpe ratio: An application of the AumannSerrano index to performance measurement', Journal of Banking 83 Finance 36(8), 2274 2284 .

Horváth, L., Kokoszka, P. and Zitikis, R. (2006), 'Testing for stochastic dominance using the weighted Mcfadden-type statistic', Journal of Econometrics 133(1), 191-205.

Knight, J. and Satchell, S. (2008), 'Testing for infinite order stochastic dominance with applications to finance, risk and income inequality', Journal of Economics and Finance 32(1), 35-46. 
Künsch, H. (1989), 'The jackknife and the bootstrap for general stationary observations', Annals of Statistics 17(3), 1217-1241.

Linton, O., Maasoumi, E. and Whang, Y.-J. (2005), 'Consistent testing for stochastic dominance under general sampling schemes', Review of Economic Studies 72(3), 735-765.

Linton, O., Song, K. and Whang, Y.-J. (2010), 'An improved bootstrap test of stochastic dominance', Journal of Econometrics 154(2), 186-202.

Liu, R. and Singh, K. (1992), Moving blocks jackknife and bootstrap capture weak dependence, in R. Lepage and L. Billard, eds, 'Exploring the Limits of Bootstrap', Wiley, New York, pp. $225-248$.

Newey, W. and McFadden, D. L. (1986), Large sample estimation and hypothesis testing, in R. F. Engle and D. McFadden, eds, 'Handbook of Econometrics', 1 edn, Vol. 4, Elsevier, chapter 36, pp. 2111-2245.

Radulović, D. (2002), On the bootstrap and empirical processes for dependent sequences, in H. Dehling, T. Mikosch and M. Sorensen, eds, 'Empirical Process Techniques for Dependent Data', Birkhauser, Berlin, pp. 345-364.

Thistle, P. D. (1993), 'Negative moments, risk aversion, and stochastic dominance', Journal of Financial and Quantitative Analysis 28(2), 301-311.

Whitmore, G. (1989), Stochastic dominance for the class of completely monotonic utility functions, in T. Fomby and T. Seo, eds, 'Studies in the Economics of Uncertainty', Springer New York, pp. $77-88$.

Yoshihara, K. (1979), 'Note on an almost sure invariance principle for some empirical processes', Yokohama Mathematical Journal 27(2), 105-110. 